\section{Rapid Improvement of Pyoderma Gangrenosum Managed with IV Methylprednisolone}

Malia B. Downing, M.D., Tony Nguyen, M.D., Aly Barland, M.D., Atieh Jibbe, M.D., Ting Wang, M.D. University of Kansas Medical Center, Department of Internal

Medicine, Division of Dermatology, Kansas City, KS

Received July 11, 2020; Accepted for publication Oct. 23, 2020; Published online Fed. 12, 2021 https: doi.org 10.17161 kijm.voll413880

\section{INTRODUCTION}

Pyoderma gangrenosum $(\mathrm{PG})$ is an uncommon cutaneous ulcerative disease. ${ }^{1-3}$ In contrast to its name, PG is neither infectious nor gangrenous. It occurs worldwide in patients of all ages, but most commonly affects women between 20 and 50 years of age. It is characterized as a neutrophilic dermatosis, though the pathogenesis of PG is not well understood. The diagnosis of pyoderma gangrenosum is one of exclusion. Of note, $\mathrm{PG}$ frequently is misdiagnosed as an infectious process resulting in unnecessary antibiotic or antifungal use. In general, it is managed with immunosuppressive therapy. Topical corticosteroids may be sufficient to treat mild and local disease, while systemic steroids are considered first line therapy for severe and more extensive disease. Antibiotics and/or antifungal therapy are not necessary unless PG is secondarily infected. We present a case of pyoderma gangrenosum managed with systemic corticosteroids. This case report reviewed the diagnosis and management of PG and highlighted the rapid improvement that can be achieved with systemic immunosuppressive.

\section{CASE REPORT}

A 55-year-old woman presented with a one-week history of several painful nodules on her hands and face that appeared abruptly. She reported similar lesions in the past that resolved without treatment. Her medical history was significant for rheumatoid arthritis for which she was not undergoing treatment due to cost. Physical exam revealed large, ulcerated nodules on the left fifth finger, right fourth finger, and left cheek near nostril (Figure 1). Given the negative cultures, and histopathology consistent with the diagnosis of pyoderma gangrenosum, the patient was started on intravenous methylprednisolone $1 \mathrm{~g}$ daily for three days, then switched to a prednisone taper of $60 \mathrm{mg}$, $40 \mathrm{mg}, 30 \mathrm{mg}, 20 \mathrm{mg}, 10 \mathrm{mg}$, then $5 \mathrm{mg}$ daily for two weeks on each dose. Trimethoprim/Sulfamethoxazole (Bactrim ${ }^{\mathrm{TM}}$ DS) was used three times weekly when prednisone was over $20 \mathrm{mg}$ daily. Within five days, the size, depth and associated pain of the ulcers had significantly improved (Figure 2). Unfortunately, the patient was lost to follow-up.

\section{DISCUSSION}

Pyoderma gangrenosum is a neutrophilic inflammatory disease that causes cutaneous ulcerations. ${ }^{3}$ There are different subtypes of PG based on clinical presentations. The most common subtype is ulcerative. It presents as a tender papule, pustule, vesicle, or nodule that rapidly expands and ulcerates with gun-metal gray undermined borders. Ulcers heal with characteristic thin, atrophic scars. It occurs most frequently on the pretibial lower extremities, although lesions may present on any area of the body including the mucosa and stomas. PG often is associated with pain out of proportion to exam. Pathergy has been reported in 20 - 30\% of cases. Patients with PG also may

\section{KANSAS JOURNAL of MEDICINE}

exhibit systemic symptoms such as fever, arthralgia, and malaise. Other subtypes include bullous, pustular, or vegetative.

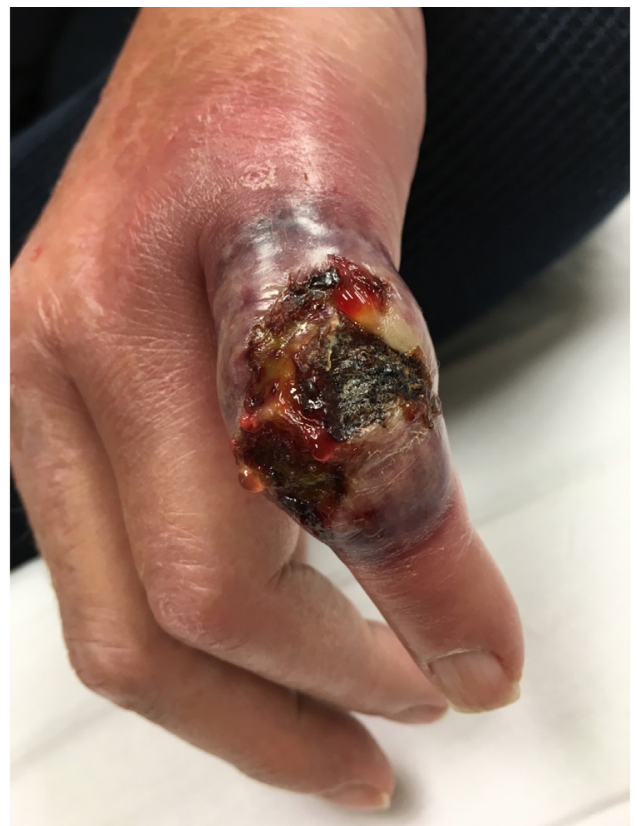

Figure 1. Ulcerated nodule of pyoderma gangrenosum on the left $5^{\text {th }}$ finger.

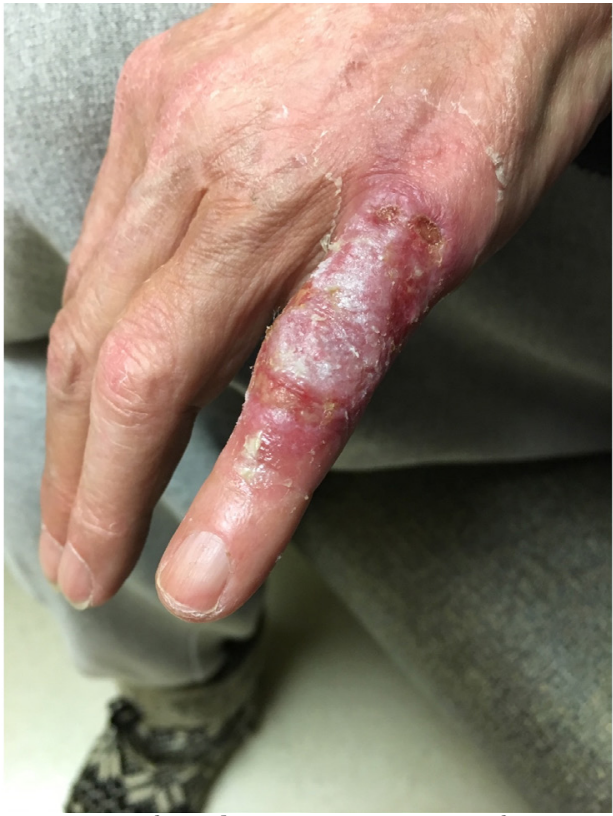

Figure 2. Improvement of pyoderma gangrenosum after IV methylprednisolone.

The diagnosis of $\mathrm{PG}$ is a diagnosis of exclusion, as there are no specific clinical, histologic or laboratory findings. ${ }^{4}$ Detailed history, complete physical exam, and skin biopsies should be performed for diagnosis and to rule out malignancy, infection, and vasculitis. Skin biopsies should be sent for both histopathologic exams and cultures including bacterial, fungal, and acid-fast bacteria. Pathology of PG should demonstrate a neutrophilic infiltrate and necrosis depending on the stage of ulceration. One recently proposed list of diagnostic criteria includes the presence of one major criterion and four out of eight minor criteria (Table 1). These criteria had a sensitivity of $80 \%$ and a specificity of $95 \%$. 


\section{KANSAS JOURNAL of MEDICINE}

PYODERMA GANGRENOSUM

continued.

Table 1. Diagnostic criteria for the diagnosis of pyoderma gangrenosum. ${ }^{5}$

\begin{tabular}{|l|l|}
\hline Major criteria (required) & $\begin{array}{l}\text { l. Biopsy of ulcer edge demonstrating } \\
\text { neutrophilic infiltrate }\end{array}$ \\
\hline Minor criteria (4 of 8 required) & 1. Exclusion of infection \\
& 2. Pathergy \\
& 3. History of inflammatory bowel \\
disease or inflammatory arthritis & 4. History of a papule, pustule, or \\
& vesicle ulcerating within four days of \\
appearing & 5. Peripheral erythema, undermining \\
& border, and tenderness at ulceration \\
& site \\
& 6. Multiple ulcerations, at least one on \\
& an anterior lower leg \\
& 7. Cribriform or wrinkles paper scar(s) \\
& at healed ulcer sites \\
& 8. Decreased ulcer size within one \\
& month of initiating immunosuppressive \\
& medications \\
\hline
\end{tabular}

Approximately $50 \%$ of cases are idiopathic while the remainder of the cases were associated with underlying systemic disease, such as inflammatory bowel disease, rheumatoid arthritis, or a hematologic disorder (IgA monoclonal gammopathy, acute myelogenous leukemia, myelodysplasia). ${ }^{1-3}$ Thus, gastrointestinal and hematologic studies are included in the recommended work-up. The most commonly associated systemic disease for PG is inflammatory bowel disease; however, only 1.5 to $5 \%$ of patients with inflammatory bowel disease will develop PG. ${ }^{6}$

The mainstay of the treatment of pyoderma gangrenosum is immunomodulatory therapy, although there is no definitive guideline for treatment. ${ }^{7-9}$ For mild and local disease, topical or intralesional corticosteroids and tacrolimus can be used. For more extensive disease, systemic immunomodulators, such as systemic corticosteroids or cyclosporine, are standard treatments, but steroid-sparing agents, such as mycophenolate mofetil or azathioprine, are often necessary during the steroid taper. Oral dapsone, and TNF-alpha inhibitors are among the medications that have been used with varying degrees of success. ${ }^{10}$ Antibiotics and/or antifungal therapy are not necessary unless PG is secondarily infected. Of note, $\mathrm{PG}$ is frequently misdiagnosed as an infectious process, resulting in unnecessary antibiotic or antifungal use. Tissue culture is commonly negative, but may yield growth of normal skin flora or demonstrate secondary superinfection. The role of surgery in treatment of $\mathrm{PG}$ is controversial due to the potential that trauma can induce or worsen $\mathrm{PG}$, also known as pathergy.

\section{REFERENCES}

${ }^{1}$ von den Driesch P. Pyoderma gangrenosum: A report of 44 cases with follow-up. Br J Dermatol 1997; 137(6):1000-1005. PMID: 9470924.

${ }^{2}$ Bennett ML, Jackson JM, Jorizzo JL, Fleischer AB Jr, White WL, Callen JP. Pyoderma gangrenosum. A comparison of typical and atypical forms with an emphasis on time to remission. Case review of 86 patients from 2 institutions. Medicine 2000; 79(1):37-46. PMID: 10670408.

3 Binus AM, Qureshi AA, Li VW, Winterfield LS. Pyoderma gangrenosum: A retrospective review of patient characteristics, comorbidities and therapy in 103 patients. Br J Dermatol 2011; 165(6):1244-1250. PMID: 21824126.

${ }^{4}$ Su WP, Davis MD, Weenig RH, Powell FC, Perry HO. Pyoderma gangrenosum: Clinicopathologic correlation and proposed diagnostic criteria. Int J Dermatol 2004; 43(11):790-800. PMID: 15533059.

${ }^{5}$ Maverakis E, Ma C, Shinkai K, et al. Diagnostic criteria of ulcerative pyoderma gangrenosum: A delphi consensus of international experts. JAMA Dermatol 2018; 154(4):461-466. PMID: 29450466.

${ }^{6}$ Farhi D, Cosnes J, Zizi N, et al. Significance of erythema nodosum and pyoderma gangrenosum in inflammatory bowel diseases: A cohort study of 2402 patients. Medicine 2008; 87(5):281-293. PMID: 18794711.

7 Ormerod AD, Thomas KS, Craig FE, et al. Comparison of the two most commonly used treatments for pyoderma gangrenosum: Results of the STOP GAP randomised controlled trial. BMJ 2015; 350:h2958. PMID: 26071094.

${ }^{8}$ Reichrath J, Bens G, Bonowitz A, Tilgen W. Treatment recommendations for pyoderma gangrenosum: An evidence-based review of the literature based on more than 350 patients. J Am Acad Dermatol 2005; 53(2):273283. PMID: 16021123.

9 Chow RK, Ho VC. Treatment of pyoderma gangrenosum. J Am Acad Dermatol 1996; 34(6):1047-1060. PMID: 8647970

${ }^{10}$ Lorincz AL, Pearson RW. Sulfapyridine and sulphone-type drugs in dermatology. Arch Dermatol 1962; 85:2-16. PMID: 14466713.

Keywords: pyoderma gangrenosum, immunosuppression, IV methylprednisolone 